\title{
A CLASS OF HOMOMORPHISM THEORIES FOR GROUPOIDS
}

\author{
by GERALD LOSEY $\dagger$ \\ (Received 25th March 1963)
}

\section{Introduction}

In a well-behaved homomorphism theory for a class $\mathfrak{b}$ of algebraic systems certain " closed objects " relative to a given $G \in \mathfrak{G}$ are distinguished which act as kernels of homomorphisms. For example, if $\mathfrak{G}$ is the class of groups then the closed objects relative to a given group $G$ are the normal subgroups of $G$; if (5) is the class of semigroups with zero element then one can devise a homomorphism theory in which the closed objects relative to a given $S \in \mathbb{G}$ are the ideals of $S[\mathrm{cf}$. Rees (3)]; in the class of groupoids one may define the closed objects relative to a given groupoid $G$ to be the congruence relations on $G$, that is, subsets $\pi \subseteq G \times G$ which are equivalence relations having the property that $\left(x_{1} y_{1}, x_{2} y_{2}\right) \in \pi$ whenever $\left(x_{1}, x_{2}\right),\left(y_{1}, y_{2}\right) \in \pi$. Given such a closed object $N$ relative to $G$ there exists a "factor" system $G / N$ and a (canonical) homomorphism $\eta: G \rightarrow G / N$ characterised by the property: If $\sigma: G \rightarrow H$ is a homomorphism with kernel $N$ then there is a unique homomorphism $\bar{\sigma}: G / N \rightarrow H$ such that $\bar{\sigma} \cdot \eta=\sigma$ and the kernel of $\bar{\sigma}$ is trivial in the sense that the kernel of $\vec{\sigma}$ is the unique smallest closed object relative to $G / N$.

In order to construct a unified homomorphism theory for groupoids which will include the examples of the preceding paragraph it is desirable that the theory include a parameter whose specification reduces the general theory to a particular one. In the theory to be constructed in this paper the parameter will be a polynomial $P(X) \in Z\left[X_{1}, \ldots, X_{n}\right], Z$ being the ring of integers.

In Section 2 we shall describe a homomorphism theory for groupoids in which the closed objects relative to a given groupoid $G$ are the ideals of the groupoid ring $Z G$. (This theory is essentially equivalent to that in which the closed objects are the congruence relations on $G$.) In Section 3 this theory will be used in defining the $P$-theory, $P=P(X)$ a polynomial. In Section 4 we shall show how the specification of $P$ gives each of the theories mentioned above.

\section{Groupoid rings and the $r$-theory}

In this section we shall construct a homomorphism theory for groupoids which we shall call the $r$-theory ( $r$ for ring).

$\dagger$ This research was supported in part by the National Science Foundation under contract NSF GP-2273. 
Let $G$ be a groupoid and $Z$ the ring of integers. Denote by $Z G$ the set of all formal sums $\Sigma_{g \in G} \alpha(g) g$ where $\alpha(g) \in Z$ and $\alpha(g)=0$ but for finitely many $g \in G$. Two sums are equal if and only if they are identical. Addition and multiplication in $Z G$ are defined in the natural manner and the resulting (not necessarily associative) ring is called the groupoid ring of $G$. The mapping $g \rightarrow \Sigma \alpha(h) h, \alpha(g)=1, \alpha(h)=0$ if $h \neq g$, is an isomorphism of $G$ into the multiplicative structure of $Z G$. We identify $g$ with its image under this isomorphism. $\dagger$

If $\phi: G \rightarrow H$ is a (groupoid) homomorphism then $\phi$ induces by linearity a ring homomorphism $\phi^{*}: Z G \rightarrow Z H$; that is,

$$
\phi^{*}\left(\Sigma_{g \in G^{\prime}} \alpha(g) g\right)=\Sigma_{g \in G^{\prime}} \alpha(g) \phi(g)=\Sigma_{h \in H}\left(\Sigma_{\phi(g)={ }_{h}} \alpha(g)\right) h .
$$

Denote the kernel of $\phi^{*}$ by $\Lambda_{\phi}$; we shall call $\Lambda_{\phi}$ the $r$-kernel of $\phi$. For each $h_{i} \in \phi(G)$ choose $g_{i} \in G$ such that $\phi\left(g_{i}\right)=h_{i}$ and set $X_{\phi}=\left\{g_{i}\right\}_{i \in I}$. Let

$$
B\left(X_{\phi}\right)=\left\{g-g_{i}: g_{i} \in X_{\phi}, \phi(g)=\phi\left(g_{i}\right), g \neq g_{i}\right\} .
$$

Then we have

Theorem 2.1. The set $B\left(X_{\phi}\right)$ is a Z-basis for $\Lambda_{\phi}$.

Proof. The $Z$-independence of $B\left(X_{\phi}\right)$ is an immediate consequence of the $Z$-independence of the elements of $G$.

Let $M$ be the $Z$-module generated by $B\left(X_{\phi}\right)$. Then, clearly, $M \subseteq \Lambda_{\phi}$. Let $\alpha=\Sigma \alpha(g) g \in \Lambda_{\phi}$. Then

$$
\begin{aligned}
& 0=\phi^{*}(\alpha)=\Sigma \alpha(g) \phi(g)=\Sigma_{i \in I} \Sigma_{\phi(g)=\phi\left(g_{i}\right.} \alpha(g) \phi\left(g_{i}\right) \\
&=\Sigma_{i \in I}\left(\Sigma_{\phi(g)}=\phi\left(g_{i}\right)\right. \\
&\alpha(g)) h_{i} .
\end{aligned}
$$

Hence, $\Sigma_{\phi(g)}=\phi\left(g_{i}\right) \alpha(g)=0$ for all $i \in I$ and so

$$
\Sigma_{\phi(g)=\phi\left(g_{i}\right)} \alpha(g) g=\Sigma_{\phi(g)=\phi\left(g_{i}\right)} \alpha(g)\left(g-g_{i}\right) .
$$

Therefore, $\alpha=\Sigma_{i \in I} \Sigma_{\phi(g)}=\phi\left(g_{i}\right) \alpha(g)\left(g-g_{i}\right) \in M$. Consequently, $\Lambda_{\phi} \subseteq M$. This completes the proof of 2.1 .

Let $\Lambda$ be any ideal of $Z G$ and let $\eta: Z G \rightarrow Z G / \Lambda$ be the canonical homomorphism. Denote by $\bar{\eta}$ the restriction of $\eta$ to $G$. Then $\bar{\eta}: G \rightarrow Z G / \Lambda$ is a homomorphism of $G$ into the multiplicative structure of $Z G / \Lambda$. Denote the image of $G$ under $\bar{\eta}$ by $G / \Lambda=\{g+\Lambda: g \in G\}$. Thus given any ideal $\Lambda$ of $Z G$ we construct the "factor" groupoid $G / \Lambda$ of $G$ by $\Lambda$. The map $\bar{\eta}: G \rightarrow G / \Lambda$ defined by $g \rightarrow g+\Lambda$ is called the canonical homomorphism. We will generally write $\eta$ for $\bar{\eta}$ if there is no danger of confusion.

Theorem 2.2. Let $\phi: G \rightarrow H$ be a homomorphism of groupoids and let $\Lambda_{\phi}$ be the $r$-kernel of $\phi$. Let $\eta: G \rightarrow G / \Lambda_{\phi}$ be the canonical homomorphism. Then there is a unique isomorphism $\bar{\phi}: G / \Lambda_{\phi} \rightarrow H$ such that $\bar{\phi} \eta=\phi$.

$\dagger$ If $G$ has a zero element $O^{\prime}$ then, using the above definition of $Z G, O^{\prime}$ is not identified with the zero element $\Sigma_{g \in G} 0 \cdot g$ of $Z G$. If it is desired to make this identification then one must reduce $Z G$ modulo the ideal $\theta=\left\{\Sigma \alpha(g) g: \alpha(g)=0\right.$ for all $\left.g \neq 0^{\prime}\right\}$. 
Proof. Define $\bar{\phi}\left(g+\Lambda_{\phi}\right)=\phi(g)$. If $g, g^{\prime} \in G$ then $\phi(g)=\phi\left(g^{\prime}\right)$ if and only if $g-g^{\prime} \in \Lambda_{\phi}$, that is, if and only if $g+\Lambda_{\phi}=g^{\prime}+\Lambda_{\phi}$. Thus $\bar{\phi}$ is both welldefined and one-one. It is easily verified to be a homomorphism. Moreover, $\bar{\phi} \eta(g)=\bar{\phi}\left(g+\Lambda_{\phi}\right)=\phi(g)$. The uniqueness of $\bar{\phi}$ follows from this last relation.

The following isomorphism theorems are also easily proved.

Theorem 2.3. Let $\phi: G \rightarrow G^{\prime}$ be a homomorphism of $G$ onto $G^{\prime}$. If $\Lambda^{\prime}$ is an ideal of $Z G^{\prime}$ then $\Lambda=\phi^{*-1}\left(\Lambda^{\prime}\right)$ is an ideal of $Z G$ containing $\Lambda_{\phi}$ and there is a unique isomorphism $\Phi: G / \Lambda \rightarrow G^{\prime} / \Lambda^{\prime}$ such that $\eta^{\prime} \phi=\bar{\phi} \eta$, where $\eta: G \rightarrow G / \Lambda$ and $\eta^{\prime}: G^{\prime} \rightarrow G^{\prime} / \Lambda^{\prime}$ are the canonical homomorphisms.

Theorem 2.4. Let $\phi: G \rightarrow H$ be a homomorphism with r-kernel $\Lambda_{\phi}$. Let $K$ be a subgroupoid of $G$ and $\tau: K \rightarrow H$ the restriction of $\phi$ to $K$. Then $\Lambda_{\tau}=\Lambda_{\phi} \cap Z K$ and, thus, there is a unique isomorphism

$$
\bar{\tau}: K / \Lambda_{\phi} \cap Z K \rightarrow H
$$

such that $\bar{\tau} \eta=\tau$, where $\eta: K \rightarrow K / \Lambda_{\phi} \cap Z K$ is the canonical homomorphism.

3. The $P$-theory.

A collection $(\mathfrak{5}$ of groupoids will be said to be complete if for every $G \in \mathfrak{G}$, (5) also contains all homomorphic images of $G$. For example, the collection of all semigroups with zero is complete but the collection of all loops is not [cf. (1)].

Let $X_{1}, \ldots, X_{n}$ be a finite set of non-associative, non-commuting indeterminates and let $P(X) \in Z\left[X_{1}, \ldots, X_{n}\right]$. The polynomial $P$ is compatible with the complete collection of groupoids $(5)$ if for every element $(k)=\left(k_{1}, \ldots, k_{n}\right)$ in $G^{n}=G \times \ldots \times G, G \in \mathfrak{G}, P\left(k_{1}, \ldots, k_{n}\right) \in Z G$. If $P$ has zero constant term then $P$ is compatible with all collections $(5$; if $P$ has a non-zero constant term then $P$ is compatible with $(5$ if and only if every groupoid $G \in \mathbb{6}$ has an identity element $1_{G}$ in which case $P(k)=a_{0} 1_{G}+a_{1} k_{1}+\ldots$.

Throughout the remainder of this section $(5$ will be a fixed complete collection of groupoids and $P$ a fixed polynomial compatible with $(\mathfrak{b}$.

Let $G \in \mathbb{G}$ and let $K$ be a non-empty subset of $G^{n}$. We define $\Delta_{P}(K)$ to be the ideal of $Z G$ generated by the elements $P(k),(k) \in K$. We define

$$
C l_{P}(K)=\left\{(g) \in G^{n}: P(g) \in \Delta_{P}(K)\right\} .
$$

If $K$ is the empty set $\varnothing$ then we set

$$
C l_{P}(\varnothing)=I_{P}(G)=\left\{(g) \in G^{n}: P(g)=0\right\} .
$$

A subset $K$ of $G^{n}$ is said to be $P$-closed if $C l_{P}(K)=K$. Note that $I_{P}(G)$ is the unique minimal $P$-closed subset of $G^{n} ; I_{P}(G)$ may be empty. We call $I_{P}(G)$ the trivial closed subset of $G^{n}$.

Theorem 3.1. The operator $\mathrm{Cl}_{P}$ satisfies the following:

(a) $K \subseteq C l_{P}(K)$;

(b) For any $K \subseteq G^{n}, C l_{P}(K)$ is P-closed; 
(c) If $\left\{K_{i}\right\}_{i \in I}$ is a collection of P-closed subsets of $G^{n}$ then $K=\bigcap_{i \in I} K_{i}$ is $P$-closed;

(d) $I_{P}(G)$ and $G^{n}$ are P-closed.

Proof. (a) and (d) follow trivially from the definition. Also from the definition we have $P(g) \in \Delta_{P}(K)$ for all $(g) \in C l_{P}(K)$. Hence, $\Delta_{P}\left(C l_{P}(K)\right)=$ $\Delta_{P}(K)$ and so $C l_{P}\left(C l_{P}(K)\right)=C l_{P}(K)$.

Let $\left\{K_{i}\right\}_{i \in I}$ be a collection of $P$-closed sets. Then $(g) \in C l_{P}\left(\bigcap K_{i}\right)$ only if $P(g) \in \Delta_{P}\left(\bigcap K_{i}\right) \subseteq \bigcap \Delta_{P}\left(K_{i}\right)$. Thus $P(g) \in \Delta_{P}\left(K_{i}\right)$ for all $i$ and hence, $(g) \in C l_{P}\left(K_{i}\right)$ $=K_{i}$ for all $i$. Thus, $(g) \in \bigcap K_{i}$. Therefore, $C l_{P}\left(\bigcap K_{i}\right) \subseteq \cap K_{i}$. Now (c) follows from (a).

Let $G, H \in(5$ and let $\sigma: G \rightarrow H$ be a homomorphism. We extend $\sigma$ to a mapping $\tilde{\sigma}: G^{n} \rightarrow H^{n}$ componentwise, that is, $\tilde{\sigma}\left(g_{1}, \ldots, g_{n}\right)=\left(\sigma\left(g_{1}\right), \ldots, \sigma\left(g_{n}\right)\right)$.

Lemma 3.2. If $\sigma: G \rightarrow H$ is a homomorphism then

for all subsets $K \subseteq G^{n}$.

$$
\sigma^{*}\left(\Delta_{P}(K)\right)=\Delta_{P}(\tilde{\sigma}(K))
$$

Proof. $\Delta_{P}(K)$ is generated by the elements $P(k),(k) \in K$, and so $\sigma^{*}\left(\Delta_{P}(K)\right)$ is generated by the elements $\sigma^{*} P(k)=P(\tilde{\sigma}(k)) \in \Delta_{P}(\sigma(K))$. Hence

$$
\sigma^{*}\left(\Delta_{P}(K)\right) \subseteq \Delta_{P}(\tilde{\sigma}(K)) \text {. }
$$

On the other hand, $\Delta_{P}(\tilde{\sigma}(K))$ is generated by the elements $P(\tilde{\sigma}(k))=\sigma^{*} P(k)$. Therefore $\Delta_{P}(\tilde{\sigma}(K)) \subseteq \sigma^{*}\left(\Delta_{P}(K)\right)$.

Using this lemma we can show that all homomorphisms are "continuous" in the following sense:

Theorem 3.3. Let $\sigma: G \rightarrow H$ be a homomorphism and let $K^{\prime}$ be a $P$-closed subset of $H$. Then $K=\tilde{\sigma}^{-1}\left(K^{\prime}\right)$ is a P-closed subset of $G$.

Proof. If $(g) \in C l_{P}(K)$ then $P(g) \in \Delta_{P}(K) \subseteq \sigma^{*-1}\left(\Delta_{P}\left(K^{\prime}\right)\right)$, by Lemma 4.2. Therefore, $\sigma^{*} P(g)=P(\tilde{\sigma}(g)) \in \Delta_{P}\left(K^{\prime}\right)$ and so $\sigma(g) \in C l_{P}\left(K^{\prime}\right)=K^{\prime}$ or

$$
(g) \in \tilde{\sigma}^{-1}\left(K^{\prime}\right)=K \text {. }
$$

Let $\sigma: G \rightarrow H$ be a homomorphism. Define the P-kernel of $\sigma$ to be the set $N=\left\{(x) \in G^{n}: \tilde{\sigma}(x) \in I_{P}\left(G^{\prime}\right)\right\}$. Since $N=\tilde{\sigma}^{-1}\left(I_{P}\left(G^{\prime}\right)\right)$ is the inverse image of a $P$-closed set, $N$ is a $P$-closed set of $G^{n}$. The homomorphism $\sigma$ is said to be a $P$-isomorphism if the $P$-kernel of $\sigma$ is $I_{P}(G)$.

If $N$ is any $P$-closed subset of $G^{n}$ then we define the $P$-factor-groupoid $(G / N)_{P}=G / \Delta_{P}(N)$. The canonical homomorphism $\eta: G \rightarrow(G / N)_{P}$ is the homomorphism $g \rightarrow g+\Delta_{p}(N)$ as defined in Section 2.

Theorem 3.4. The P-kernel of the natural homomorphism $\eta: G \rightarrow(G / N)_{P}$ is N. Moreover,

$$
I_{p}\left((G / N)_{P}\right)=\left\{\left(\bar{g}_{1}, \ldots, \bar{g}_{n}\right): \bar{g}_{i}=g_{i}+\Delta_{P}(N),\left(g_{i}\right) \in N\right\} .
$$

Proof. $I\left(\left({ }_{P} G / N\right)_{P}\right)$ is the set of all $\left(\bar{g}_{1}, \ldots, \bar{g}_{n}\right), \bar{g}_{i}=g_{i}+\Delta_{P}(N)$, such that $P\left(\bar{g}_{1}, \ldots, \bar{g}_{n}\right)=P\left(g_{1}, \ldots, g_{n}\right)+\Delta_{P}(N)=\Delta_{P}(N)$. Thus, $(g) \in G^{n}$ is in the $P$-kernel of $\eta$ if and only if $P(g) \in \Delta_{P}(N)$, that is, if and only if $(g) \in N$. 


\section{CLASS OF HOMOMORPHISM THEORIES FOR GROUPOIDS 133}

Theorem 3.5. Let $\sigma: G \rightarrow H$ be a homomorphism with P-kernel $N$. Then there exists a unique P-isomorphism $\bar{\sigma}:(G / N)_{P} \rightarrow H$ such that $\bar{\sigma} \eta=\sigma$, where $\eta: G \rightarrow(G / N)_{P}$ is the canonical homomorphism.

Proof. Define $\bar{\sigma}:(G / N)_{P} \rightarrow H$ by $g+\Delta_{P}(N) \rightarrow \sigma(g)$. If $g+\Delta_{P}(N)=g^{\prime}+\Delta_{P}(N)$ then $g-g^{\prime} \in \Delta_{P}(N)$ and so

$$
\sigma(g)-\sigma\left(g^{\prime}\right)=\sigma\left(g-g^{\prime}\right) \in \sigma^{*}\left(\Delta_{P}(N)\right)=\Delta_{P}(\tilde{\sigma}(N))=\Delta_{P}\left(I_{P}(H)\right)=\{0\}
$$

since $P(h)=0$ for all $(h) \in I_{P}(H)$. Therefore, $\sigma(g)=\sigma\left(g^{\prime}\right)$ and so $\bar{\sigma}$ is well defined. It is clearly a homomorphism. An element $(\bar{g}) \in(G / N)_{P}^{n}$ belongs to the $P$-kernel of $\bar{\sigma}$ if and only if

$$
\left(\bar{\sigma}\left(\bar{g}_{1}\right), \ldots, \bar{\sigma}\left(\bar{g}_{n}\right)\right)=\left(\sigma\left(g_{1}\right), \ldots, \sigma\left(g_{n}\right)\right)=\sigma\left(g_{1}, \ldots, g_{n}\right) \in I_{P}(H),
$$

that is, if and only if $(g) \in N$. Thus, by $4.4,(\bar{g})$ is in the kernel of $\bar{\sigma}$ if and only if $(\bar{g}) \in I_{P}\left((G / N)_{P}\right)$. Hence $\bar{\sigma}$ is a $P$-isomorphism. Moreover

$$
\bar{\sigma} \eta(g)=\bar{\sigma}\left(g+\Delta_{P}(N)\right)=\sigma(g) .
$$

The uniqueness of $\bar{\sigma}$ follows from this relation.

The property of theorem 3.5 characterises the $P$-factor-group up to isomorphism.

Theorem 3.6. Let $G$ be a groupoid and $N$ a P-closed subset of $G^{n}$. Let $K$ be a groupoid and $\mu: G \rightarrow K$ a homomorphism with P-kernel $N$. Suppose that for every homomorphism $\phi: G \rightarrow H$ with $P$-kernel $N$ there exists a $P$-isomorphism $\phi^{\prime}$ : $K \rightarrow H$ such that $\phi^{\prime} \mu=\phi$. Then $K$ and $(G / N)_{P}$ are isomorphic.

Proof. By 3.5 there exists a homomorphism $\bar{\mu}:(G / N)_{P} \rightarrow K$ such that $\bar{\mu} \eta=\mu$. By the hypothesis, there exists a homomorphism $\eta^{\prime}: K \rightarrow(G / N)_{P}$ such that $\eta^{\prime} \mu=\eta$. Hence $\left(\bar{\mu} \eta^{\prime}\right) \mu=\mu$ and $\left(\eta^{\prime} \bar{\mu}\right) \eta=\eta$. Thus $\bar{\mu} \eta^{\prime}$ is the identity mapping on $K$ and $\eta^{\prime} \bar{\mu}$ is the identity mapping on $(G / N)_{P}$. Therefore $\bar{\mu}$ and $\eta^{\prime}$ are inverse isomorphisms.

The following " isomorphism" theorems can be proved in a manner similar to that of 3.5 .

Theorem 3.7. Let $\sigma: G \rightarrow G^{\prime}$ be a homomorphism of $G$ onto $G^{\prime}$ with P-kernel $N$. Then there is a one-one correspondence between the P-closed subsets $H^{\prime}$ of $G^{\prime n}$ and the $P$-closed subsets $H$ of $G^{n}$ containing $N$ given by $H=\tilde{\sigma}^{-1}\left(H^{\prime}\right)$. If $H$ and $H^{\prime}$ are corresponding $P$-closed subsets then there exists a unique $P$-isomorphism $\bar{\sigma}:(G / H)_{P} \rightarrow\left(G^{\prime} / H^{\prime}\right)_{P}$ such that $\eta^{\prime} \sigma=\bar{\sigma} \eta$, where $\eta: G \rightarrow(G / H)_{P}$ and $\eta^{\prime}: G^{\prime} \rightarrow$ $\left(G^{\prime} / H^{\prime}\right)_{P}$ are the canonical homomorphisms.

Theorem 3.8. Let $\sigma: G \rightarrow H$ be a homomorphism with P-kernel $N$. Let $K$ be a subgroupoid of $G$ and let $\tau: K \rightarrow H$ be the restriction of $\sigma$ to $K$. Then the $P$-kernel of $\tau$ is $N \cap K^{n}$ and, thus, there exists a unique P-isomorphism

$$
\bar{\tau}:\left(K / N \cap K^{n}\right)_{P} \rightarrow H
$$

such that $\bar{\tau} \eta=\tau$, where $\eta: K \rightarrow\left(K / N \cap K^{n}\right)_{P}$ is the canonical homomorphism. 


\section{Examples}

I. Let $\mathfrak{G}$ be the collection of all groups and let $P(X)=X-1$. Then the $P$-closed subsets of a group $G$ will be subsets of $G^{1}=G$. We claim that the $P$-closed subsets of $G$ are precisely the normal subgroups of $G$.

Suppose $H \subseteq G$ and $H$ is $P$-closed. Then $x, y \in H$ implies that $x-1$, $y-1 \in \Delta_{P}(H)$ and thus that $x-1,-y^{-1}(y-1)=y^{-1}-1 \in \Delta_{P}(H)$. Therefore, $x y^{-1}-1=(x-1)\left(y^{-1}-1\right)+(x-1)+\left(y^{-1}-1\right) \in \Delta_{P}(H)$ and so $x y^{-1} \in H$. Thus $H$ is a subgroup of $G$. Moreover, for any $x \in H, z \in G$ we have

$$
z^{-1} x z-1=z^{-1}(x-1) z \in \Delta_{P}(H) \text {. }
$$

Therefore, $H$ is a normal subgroup of $G$. Conversely, suppose $H$ is a normal subgroup of $G$ and $G=G / H$. Then the kernel of the homomorphism $Z G \rightarrow Z \bar{G}$ defined by $\Sigma \alpha(g) g \rightarrow \Sigma \alpha(g) \bar{g}, \bar{g}=g H$, is precisely $\Delta_{P}(H)$. [This follows from 2.2; see also Jennings, (2).] Therefore, if $g \in C l_{P}(H)$ then $g-1 \in \Delta_{P}(H)$ and so $\bar{g}=\overline{1}$, that is $g \in H$. Hence $H=C l_{\mathrm{P}}(H)$.

The trivial $P$-closed subset of $G$ is $\{1\}$ and it is easy to see that every $P$ isomorphism is an isomorphism. By 3.6, $(G / H)_{P} \cong G / H$ and this isomorphism is given by $g+\Delta_{P}(H) \leftrightarrow g H$.

II. Let $\mathscr{S}$ be the collection of all semigroups with 0 and let $Q(X)=X$. The $Q$-closed sets are subsets of $S^{1}=S$. We claim that the $Q$-closed subsets of $S$ are precisely the ideals of $S$.

Suppose $J$ is a $Q$-closed subset of $S$. Then $x \in J$ if and only if $x \in \Delta_{Q}(J)$. Thus if $x \in J$ and $y \in S$ then both $y x$ and $x y$ belong to $\Delta_{Q}(J)$ and, hence, to $J$. Therefore, $J$ is an ideal of $S$. Conversely, suppose $J$ is an ideal of $S$. Then it is clear that $\Delta_{Q}(J)$ is the set of all finite sums $\Sigma_{x \in J} n(x) x$ where $n(x) \in Z$. If $y \in C l_{Q}(J)$ then $y \in S$ and $y=\Sigma_{x \in J} n(x) x$. By the linear independence of the elements of $S$ in $Z S$, this implies $y=x$ for some $x \in J$. Consequently, $C l_{Q}(J)=J$ and $J$ is $Q$-closed.

Let $S$ be a semigroup with $\overline{0}$ and $J$ an ideal of $S$. Then

$$
(S / J)_{Q}=\left\{x+\Delta_{Q}(J): x \in S\right\} .
$$

Let $\bar{x}=x+\Delta_{Q}(J)$. Then $\bar{x}=\overline{0}$ if and only if $x \in \Delta_{Q}(J)$, that is, if and only if $x \in J$. If $x, y \in S$ then $\bar{x}=\bar{y}$ if and only if $x-y \in \Delta_{Q}(J)$. But this can happen if and only if $x=y$ or $x$ and $y \in J$, that is, $\bar{x}=\bar{y}$ implies $x=y$ or $\bar{x}=\bar{y}=\overline{0}$. Thus, under the canonical homomorphism, the elements of $J$ are mapped onto $\overline{0}$ and the elements $x \in S-J$ are mapped onto distinct elements $\bar{x}=\overline{0}$. This is precisely the definition of the Rees quotient of $S$ by $J$ [cf. Rees (3)].

III. Let $\mathscr{M}$ be the class of all groupoids and $R(X, Y)=X-Y$. The $R$-closed sets will be subsets of $G \times G, G \in \mathscr{M}$. The trivial $R$-closed subset will be the diagonal: $I_{R}(G)=\{(g, g): g \in G\}$. We claim that the $R$-closed subsets of $G \times G$ are precisely the congruence relations in $G \times G$.

Suppose $\pi \subseteq G \times G$ is $R$-closed. Clearly $(x, x) \in \pi$ for all $x \in G$ since $I_{R}(G)$ is contained in all $R$-closed sets. If $(x, y) \in \pi$ then $R(y, x)=-R(x, y) \in \Delta_{R}(\pi)$ and so $(y, x) \in \pi$. If $(x, y),(y, z) \in \pi$ then

$$
(x-y)+(y-z)=(x-z)=R(x, z) \in \Delta_{R}(\pi)
$$




\section{CLASS OF HOMOMORPHISM THEORIES FOR GROUPOIDS 135}

and so $(x, z) \in \pi$. Thus $\pi$ is an equivalence relation. Let $\left(x_{1}, y_{1}\right),\left(x_{2}, y_{2}\right) \in \pi$. Then $\left(x_{1}-y_{1}\right) x_{2}+y_{1}\left(x_{2}-y_{2}\right)=x_{1} x_{2}-y_{1} y_{2} \in \Delta_{R}(\pi)$ and so $\left(x_{1} x_{2}, y_{1} y_{2}\right) \in \pi$. Therefore $\pi$ is a congruence relation in $G \times G$.

Conversely, let $\pi$ be a congruence relation in $G \times G$. Let $\mathrm{G}^{\prime}$ be the groupoid of congruence classes, $G^{\prime}=G / \pi$ and let $\tilde{\pi}: G \rightarrow G^{\prime}$ be the natural mapping, that is, $\tilde{\pi}(g)$ is the congruence class containing $g$. Then $\tilde{\pi}^{*}: Z G \rightarrow Z G^{\prime}$ has kernel $\Lambda_{\tilde{x}}$ which is spanned over $Z$ by the elements $x_{i}-y_{i},\left(x_{i}, y_{i}\right) \in \pi$. Since $\Delta_{R}(\pi)$ is spanned over $Z G$ by the elements $x_{i}-y_{i},\left(x_{i}, y_{i}\right) \in \pi$, it follows that

$$
\Delta_{R}(\pi)=\Lambda_{\tilde{\pi}} \text {. }
$$

Hence every element of $\Delta_{R}(\pi)$ can be expressed in the form $\Sigma n_{i}\left(x_{i}-y_{i}\right)$ where $\left(x_{i}, y_{i}\right) \in \pi, n_{i} \in Z$. Since $\left(x_{i}, y_{i}\right) \in \pi$ implies $\left(y_{i}, x_{i}\right) \in \pi$ we can further assume that every element of $\Delta_{R}(\pi)$ can be written $\Sigma\left(x_{i}-y_{i}\right)$ with $\left(x_{i}, y_{i}\right) \in \pi$ (with repetitions allowed). Suppose that in such an expression $y_{i}=x_{j}$ for some $i$ and $j$; then we can replace $\left(x_{i}-y_{i}\right)+\left(x_{j}-y_{j}\right)$ by $x_{i}-y_{j}$ since $\left(x_{i}, y_{i}\right),\left(x_{j}, y_{j}\right) \in \pi$ implies $\left(x_{i}, y_{j}\right) \in \pi$. Thus every element of $\Delta_{R}(\pi)$ can be written $\Sigma\left(x_{i}-y_{i}\right)$ where $\left(x_{i}, y_{i}\right) \in \pi$ and no $y_{i}=x_{j}$. Now suppose $(x, y) \in C l_{R}(\pi)$; then

$$
x-y=\Sigma\left(x_{i}-y_{i}\right) \in \Delta_{R}(\pi) \text {. }
$$

If the sum on the right included more than one term then, in order that the appropriate cancellations occur, we would have $y_{1}=x_{j}$ for some $j>1$. But this cannot occur. Therefore $x-y=x_{1}-y_{1}$ and so $x=x_{1}$ and $y=y_{1}$. Thus $(x, y)=\left(x_{1}, y_{1}\right) \in \pi$. Hence $\pi$ is $R$-closed.

The isomorphism $G / \pi \cong(G / \pi)_{R}$ is given by $\tilde{\pi}(g) \leftrightarrow g+\Delta_{R}(\pi)$.

\section{REFERENCES}

(1) R. H. BRUCK, A Survey of Binary Systems (Berlin, 1958).

(2) S. A. Jennings, The group ring of a class of infinite nilpotent groups, Canadian J. Math., 7 (1955), 169-187.

(3) D. Rees, On semigroups, Proc. Cambridge Phil. Soc., 36 (1940), 387-400.

THE UNIVERSITY OF WISCONSIN 